\title{
Fabry-Pérot Cavity Based on Hollow-Core Ring Photonic Crystal Fiber for Pressure Sensing
}

\author{
Marta S. Ferreira, Joerg Bierlich, H. Lehmann, Kay Schuster, Jens Kobelke, \\ José Luís Santos, and Orlando Frazão
}

\begin{abstract}
An interferometric Fabry-Pérot cavity based on hollow-core ring photonic crystal fiber (HCR-PCF) for pressure sensing is proposed. The sensing head is formed by splicing a small section of HCR-PCF to standard single mode fiber. The spectral response depends on the cavity length due to the geometry of the HCR-PCF. The sensing head is subjected to methane pressure variations, where it exhibits a sensitivity of $0.82 \mathrm{~nm} / \mathrm{MPa}$. Its response to nitrogen pressure variation is also studied. The sensing head's intrinsic sensitivity to the nitrogen refractive index variations inside the hollow-core is also estimated. Finally, temperature measurement is performed and a sensitivity of $3.77 \mathrm{pm} /{ }^{\circ} \mathrm{C}$ is obtained for temperatures below $200{ }^{\circ} \mathrm{C}$.
\end{abstract}

Index Terms-Interferometry, optical fiber sensor, photonic crystal fiber.

\section{INTRODUCTION}

$\mathbf{T}$ HE USE of Fabry-Pérot (FP) cavities for optical sensing has been widely studied over the last years. Besides their simple configuration and high sensitivity, they are usually compact, reliable and stable devices [1]. Several combinations of fiber elements have been studied, and different physical, chemical and biological parameters were characterized.

In 2007, Rao et al [2] proposed the splicing of a hollow-core photonic crystal fiber (HC-PCF) section between two single mode fibers (SMF28). This was the first fiber optic in-line etalon reported. It was tested in strain, where an accuracy better than $\sim 5 \mu \varepsilon$ was achieved. In the same year, the fabrication of a FP cavity on a PCF using laser micromachining was reported [3]. The sensing head presented linear response to strain over several operating temperatures. In 2009, Villatoro et al [1] presented a spherical microscopic FP cavity. It was also tested for strain and temperature, presenting low thermal sensitivity and a strain sensitivity of $2.7 \mathrm{pm} / \mu \varepsilon$. Due to their

Manuscript received July 15, 2012; revised September 3, 2012; accepted September 28, 2012. Date of publication October 10, 2012; date of current version November 20, 2012. This work was supported in part by the Fundação para a Ciência e Tecnologia under Grant SFRH/BD/76965/2011.

M. S. Ferreira and J. L. Santos are with INESC Porto, Porto 4169-007, Portugal, and also with the Faculdade de Ciências da Universidade do Porto, Porto 4169-007, Portugal (e-mail: msaf@inescporto.pt; josantos@fc.up.pt).

J. Bierlich, H. Lehmann, K. Schuster, and J. Kobelke are with Institute of Photonic Technology (IPHT) Jena, Jena 07745, Germany (e-mail: joerg.bierlich@ipht-jena.de; kay.schuster@ipht-jena.de; jens.kobelke@ ipht-jena.de).

O. Frazão is with INESC Porto, Porto 4169-007, Portugal (e-mail: ofrazao@inescporto.pt).

Color versions of one or more of the figures in this letter are available online at http://ieeexplore.ieee.org.

Digital Object Identifier 10.1109/LPT.2012.2222368 spectral characteristics, FP cavities can also be used as optical filters. A tunable FP filter based on a photonic bandgap fiber and a microfiber has been proposed, thus producing a narrow-linewidth single longitudinal mode laser [4]. In 2004, Xiao et al [5] presented an air cavity FP interferometer to measure gas-pressure change and used two different interrogation setups. For nitrogen $\left(\mathrm{N}_{2}\right)$ measurements, a sensitivity of $34.9 \mathrm{~nm} / \mathrm{psi}(0.24 \mathrm{~nm} / \mathrm{MPa})$ was reported. In 2010, Deng et al [6] proposed a configuration capable of measuring the refractive index of both liquids and gases. The sensing head was formed by a section of hollow core fiber spliced between a SMF28 and a PCF. A wavelength sensitivity of $1639 \mathrm{~nm} / \mathrm{RIU}$ for carbon dioxide $\left(\mathrm{CO}_{2}\right)$ measurements was reported. More recently, Duan et al [7] studied the response of a FP cavity to air refractive index variations. The cavity was formed by splicing a short section of SMF28 between two sections of SMF28 with a large lateral offset. The sensing head presented low thermal sensitivity and an air refractive index sensitivity of $\sim 1540 \mathrm{~nm} / \mathrm{RIU}$. Methane $\left(\mathrm{CH}_{4}\right)$ gas pressure is of most importance in safety monitoring in energy exploration, as well as in environmental applications. Most of the works published on this subject are based in absorption spectroscopy [8]. $\mathrm{CH}_{4}$ presents two absorption lines $(1.33 \mu \mathrm{m}$ and $1.65 \mu \mathrm{m})$ that correspond to the fiber operation region with low losses. Several works have been published using distributed feedback lasers [9]. Besides, with the emerging of the photonic band gap fiber (PBGF), new solutions have been reported [10].

In this letter, a Fabry-Pérot cavity based on a large hollowcore ring photonic crystal fiber (HCR-PCF) with an open air-hole is proposed. The FP cavity is tested for gas pressure sensing with constant concentration. The sensing head is also subjected to temperature variations.

\section{EXPERIMENTAL RESULTS}

The experimental setup, shown in Figure 1, was constituted by a broadband optical source with a spectral bandwidth of $100 \mathrm{~nm}$ at $1550 \mathrm{~nm}$, an optical spectrum analyzer with a resolution of $0.5 \mathrm{~nm}$ and an optical circulator to measure the FP cavity spectral response in reflection. This FP cavity was created by splicing a small section of the large hollow-core ring PCF (HCR-PCF) to standard single mode fiber (SMF28). The splice was mainly applied in the SMF28 region to avoid collapsing the holes in the HCR-PCF. A manual program of the splice machine was used, according to the details described in reference [11]. Figure 1 also presents the cross section image of the HCR-PCF when observed at the microscope. This fiber 


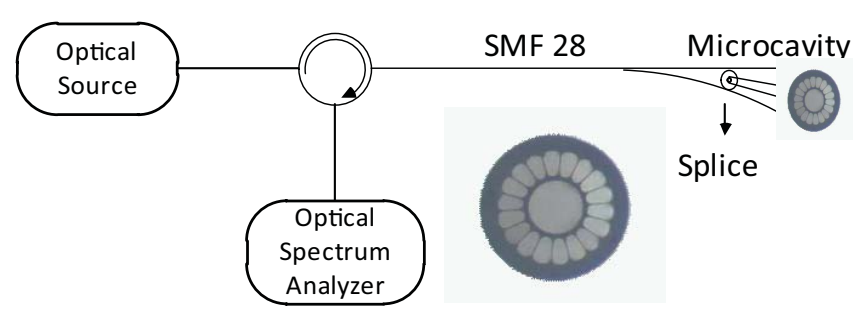

Fig. 1. Scheme of the experimental setup and image of the large hollow-core PCF cross section.

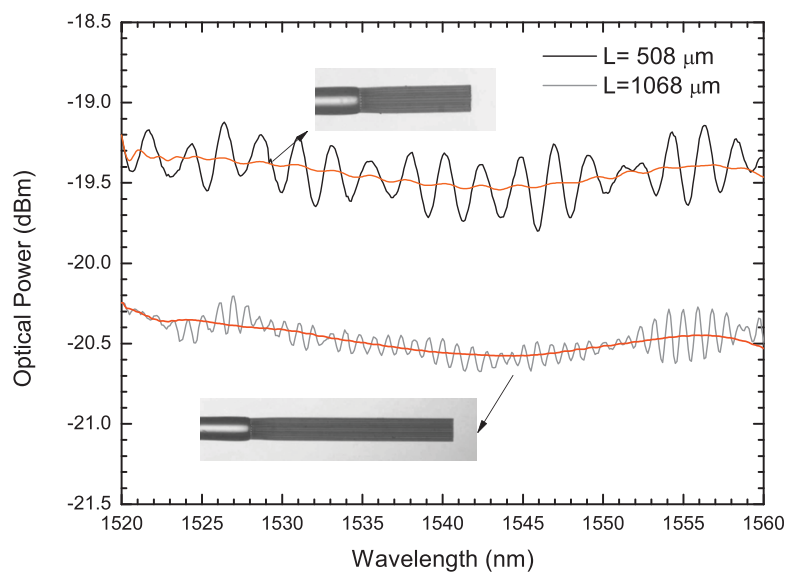

Fig. 2. Spectra of two different sensing heads evidencing the interferometric behavior and the optical losses.

presents a large hollow core, with a diameter of $44.4 \mu \mathrm{m}$ and several petal shaped holes with an azimuthal diameter of $24.4 \mu \mathrm{m}$. In between these two structures, there is a $3.1 \mu \mathrm{m}$ thick silica ring. The cavity is formed when light travelling from the SMF end face excites the PCF ring. Afterwards, it is reflected due to the HCR-PCF end face cleavage, being recoupled to the SMF. The spectral response depends on the FP cavity length. For smaller sensing heads (lower than $150 \mu \mathrm{m}$ ) the optical path is very small and the ring is not excited. This occurs since the numerical aperture (0.14 for SMF28) is out of the cavity length. When the cavity length is longer than $150 \mu \mathrm{m}$, the ring is excited and interference is generated. Figure 2 presents the spectra of two different sensing head lengths, showing in particular that the losses increase with the sensing head length. The average spectral behavior is similar in both cases. As it can be seen from this figure, for the smaller FP, the visibility is $\sim 0.045$, whilst for the larger one it is $\sim 0.020$. Besides this decrease of $\sim 56 \%$ in the visibility, the intensity losses are increased by $\sim 1 \mathrm{~dB}$. The interferometric period for the shorter sensing head $(508 \mu \mathrm{m})$ is $10 \mathrm{~nm}$ while for the longer one $(1068 \mu \mathrm{m})$ it is $4 \mathrm{~nm}$. The interferometric patterns shows some beating, suggesting interference of more than two waves, which means the guidance in the silica ring is multimode. Anyway, the small beating effect combined with the reduced ring thickness indicates few modes are guided by the structure. The group effective refractive index where light is travelling can be estimated through the expression

$$
n=\frac{\lambda_{1} \lambda_{2}}{2 L\left(\lambda_{2}-\lambda_{1}\right)}
$$

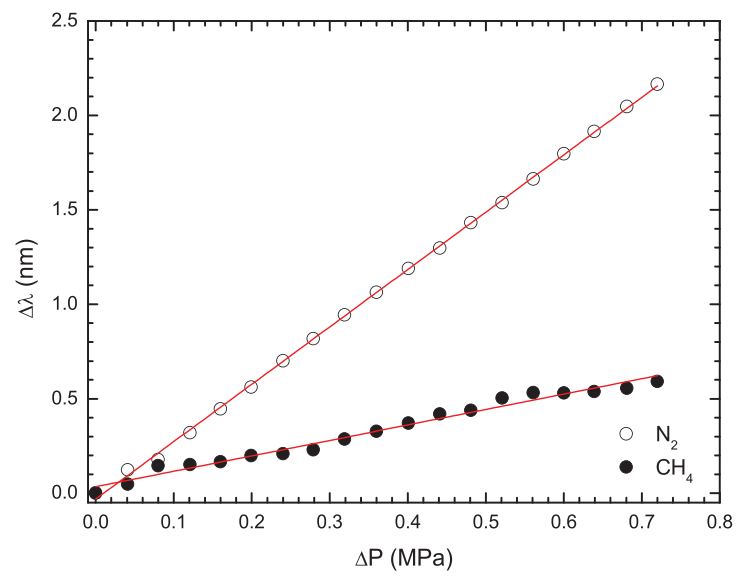

Fig. 3. Sensing head response to pressure variations of two different gases $(L=508 \mu \mathrm{m})$.

Where $\mathrm{L}$ is the cavity length and $\lambda_{1}$ and $\lambda_{2}$ are the peak wavelengths of two adjacent fringes. For the shorter cavity we obtain $\mathrm{n} \sim 1.17$, while for the longer one it results into $\mathrm{n} \sim 1.28$. These values come from the combination of three contributions: the propagation in air when the light exits the SMF28 core up to reach the silica ring; propagation in the silica ring; evanescent field in air of the light that propagates in this ring. The relative weight of the first contribution is larger for the shorter cavity, reason why the effective refractive index has a smaller value in this case. The pressure sensing of this structure relies on the gas refractive index variation induced by pressure changes, combined with its interaction with the evanescent optical field that propagates in the hollowcore ring. Therefore, if this interaction occurs on a longer length the sensitivity will increase. However, the loss will also increase and the interferometric visibility will decrease, as the data shown in Figure 2 reveals. In view of this a compromise is needed, which is fairly achieved with the chosen cavity length of $508 \mu \mathrm{m}$. To measure $\mathrm{CH} 4$ pressure, the $508 \mu \mathrm{m}$ long sensing head was placed inside a sealed chamber, with a gas input and a vacuum purge output. When the pressure changes, a shift of the interferometric fringes occurs, as can be observed in Figure 3. The sensitivity obtained was $0.82 \mathrm{~nm} / \mathrm{MPa}$, for a pressure range of $0.75 \mathrm{MPa}$. Measurements with a noble gas atmosphere were also done, in this case, $\mathrm{N}_{2}$ was used with the same pressure range and the sensitivity achieved was $3.04 \mathrm{~nm} / \mathrm{MPa}$ (Figure 3) These results indicate: i) it is possible to measure pressure with this sensing head through variation of the refractive index of the gas inside the fiber hollowcore; ii) the sensitivity to pressure variations is gas dependent, which means the possibility to identify the gas present in the interferometric cavity by analyzing the response of the sensor to pressure changes. The intrinsic sensitivity of the sensing head to refractive index changes of the gas inside the hollowcore can be determined from the results showed in Figure 3 for $\mathrm{N}_{2}$. According to references [6], [12], the refractive index of air in a closed cylinder is linearly related to pressure by equation (2):

$$
n=1+2.8793 \times 10^{-9} P /(1+0.003671 \times T)
$$




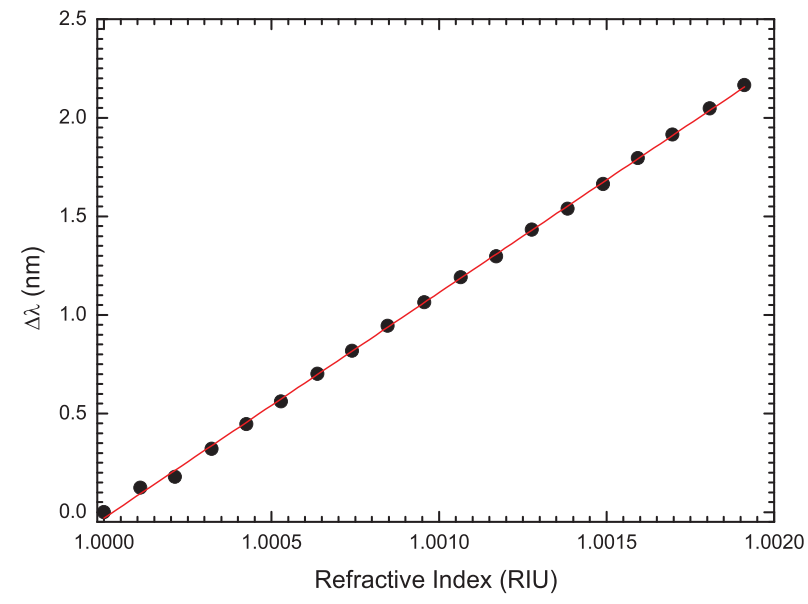

Fig. 4. Sensing head response to refractive index variations $(L=508 \mu \mathrm{m})$.

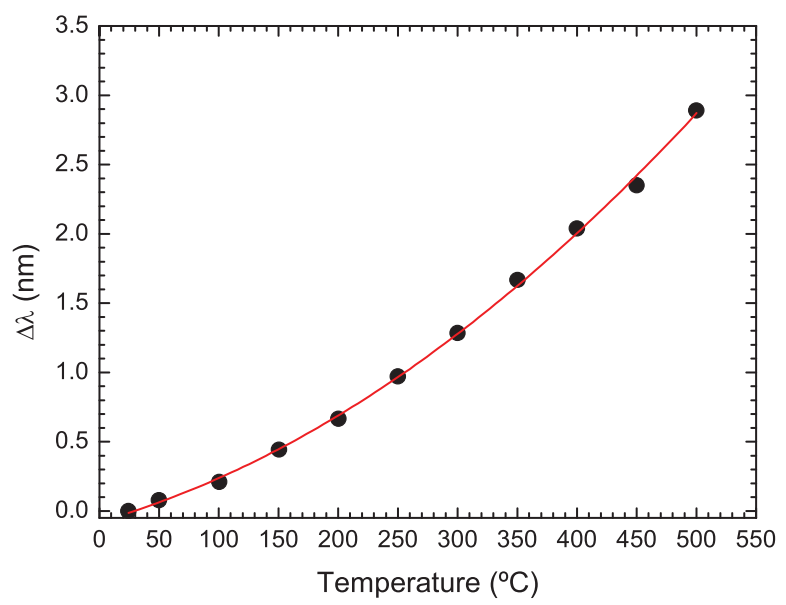

Fig. 5. Temperature response of the 508- $\mu \mathrm{m}$-long sensing head.

Where $\mathrm{n}$ is the refractive index, $\mathrm{P}$ the pressure (in $\mathrm{Pa}$ ) and $\mathrm{T}$ is the temperature $\left({ }^{\circ} \mathrm{C}\right)$. Thus, applying this equation to the experimental results obtained for the case of $\mathrm{N}_{2}$ in the cavity (an approximation but justifiable considering air is $78 \% \mathrm{~N}_{2}$ ), the relationship between the wavelength shift and refractive index variations can be obtained, as shown in Figure 4. From this data a sensitivity of $1144.2 \mathrm{~nm} / \mathrm{RIU}$ was obtained. The sensing head was also characterized in temperature, an important information not only in face of the possibility of applying this structure to measure temperature, but also for compensation of its cross-sensitivity effects when the sensor is used for pressure sensing. The measurements were done by placing the same sensing head inside a tubular oven, with an error associated to temperature reading smaller than $0.1{ }^{\circ} \mathrm{C}$. By subjecting the sensing head to a temperature variation of $\sim 475{ }^{\circ} \mathrm{C}$, a variation in wavelength was observed, according to Figure 5. This behavior can be well adjusted to the second order polynomial:

$$
\Delta \lambda=6.878 \times 10^{-6} T^{2}+0.0025 T-0.077
$$

Two different linear regions can be considered, for lower and higher temperatures. The sensitivity obtained for temperatures below $200{ }^{\circ} \mathrm{C}$ was $\sim 3.8 \mathrm{pm} /{ }^{\circ} \mathrm{C}$, whereas for temperatures above that value the sensitivity achieved was $\sim 7.5 \mathrm{pm} /{ }^{\circ} \mathrm{C}$. These values arise from the thermal expansion and thermooptic effects, which give same signal contributions with the last one having larger impact.

\section{CONCLUSION}

In conclusion, a FP cavity based on large hollow-core photonic crystal fiber spliced to single mode fiber was presented. The cavity is easy to manufacture and the results are reproducible. The sensing head was subjected to pressure variations with $\mathrm{CH}_{4}$ and $\mathrm{N}_{2}$ atmospheres. For the former case, it presented a sensitivity of $0.82 \mathrm{~nm} / \mathrm{MPa}$. The intrinsic sensitivity of the sensing head to refractive index changes of the gas inside the hollow-core was estimated to be $1144.2 \mathrm{~nm} / \mathrm{RIU}$. Finally, the sensing head was subjected to temperature variations, where it presented a non-linear behavior.

\section{REFERENCES}

[1] J. Villatoro, V. Finazzi, G. Coviello, and V. Pruneri, "Photonic-crystalfiber-enabled micro-Fabry-Pérot interferometer," Opt. Lett., vol. 34, no. 16, pp. 2441-2443, 2009.

[2] Y. J. Rao, T. Zhu, X. C. Yang, and D. W. Duan, "In-line fiber-optic etalon formed by hollow-core photonic crystal fiber," Opt. Lett., vol. 32, no. 18, pp. 2662-2664, 2007.

[3] Z. L. Ran, Y. J. Rao, H. Y. Deng, and X. Liao, "Miniature in-line photonic crystal fiber etalon fabricated by $157 \mathrm{~nm}$ laser micromachining," Opt. Lett., vol. 32, no. 21, pp. 3071-3073, 2007.

[4] X. Wang, T. Zhu, L. Chen, and X. Bao, "Tunable Fabry-Pérot filter using hollow-core photonic bandgap fiber and micro-fiber for a narrow-linewidth laser," Opt. Express, vol. 19, no. 10, pp. 9617-9625, 2011.

[5] G. Z. Xiao, A. Adnet, Z. Zhang, Z. Lu, and C. Grover, "Fiber-optic Fabry-Pérot interferometric gas-pressure sensors embedded in pressure fittings," Microw. Opt. Technol. Lett., vol. 42, no. 6, pp. 486-489, 2004.

[6] M. Deng, C.-P. Tang, T. Zhu, Y.-J. Rao, L.-C. Xu, and M. Han, "Refractive index measurement using photonic crystal fiber-based Fabry-Pérot interferometer," Appl. Opt., vol. 49, no. 9, pp. 1593-1598, 2010.

[7] D. W. Duan, Y. J. Rao, and T. Zhu, "High sensitivity gas refractometer based on all-fiber open-cavity Fabry-Perot interferometer formed by large lateral offset splicing," J. Opt. Soc. Amer. B, vol. 29, no. 5, pp. 912-915, 2012.

[8] B. Culshaw, G. Stewart, F. Dong, C. Tandy, and D. Moodie, "Fibre optic techniques for remote spectroscopic methane detection-from concept to system realization," Sensors Actuat. B, vol. 51, nos. 1-3, pp. 25-37, 1998.

[9] G. Stewart, A. Mencaglia, W. Philp, and W. Jin, "Interferometric signals in fiber optic methane sensors with wavelength modulation of the DFB laser source," J. Lightw. Technol., vol. 16, no. 1, pp. 43-53, Jan. 1998.

[10] T. Ritari, et al., "Gas sensing using air-guiding photonic bandgap fibers," Opt. Express, vol. 12, no. 17, pp. 4080-4087, 2004.

[11] O. Frazão, J. P. Carvalho, and H. M. Salgado, "Low loss splice in a microstructured fibre using a conventional fusion splicer," Microw. Opt. Technol. Lett., vol. 46, no. 2, pp. 172-174, 2005.

[12] K. P. Birch and M. J. Downs, "An updated Edlén equation for the refractive index of air," Metrologia, vol. 30, no. 3, pp. 155-162, 1993. 